\title{
Signaling in Regulation of Podocyte Phenotypes
}

\author{
Peter Y. Chuang ${ }^{\mathrm{a}}$ John C. He $\mathrm{He}^{\mathrm{a}-\mathrm{c}}$ \\ Departments of a Medicine and ${ }^{b}$ Pharmacology and Systems Therapeutics, Mount Sinai School of Medicine, \\ New York, N.Y., and 'James J. Peters Veterans Affairs Medical Center, Bronx, N.Y., USA
}

\section{Key Words}

Podocyte $\cdot$ Apoptosis $\cdot$ Actin $\cdot$ Signal transduction •

Glomerular nephropathy

\begin{abstract}
The kidney podocyte is a terminally differentiated and highly specialized cell. The function of the glomerular filtration barrier depends on the integrity of the podocyte. Podocyte injury and loss have been observed in human and experimental models of glomerular diseases. Three major podocyte phenotypes have been described in glomerular diseases: effacement, apoptosis, and proliferation. Here, we highlight the signaling cascades that are responsible for the manifestation of these pathologic phenotypes. The integrity of the podocyte foot process is determined by the interaction of nephrin with proteins in the slit diaphragm complex, the regulation of actin dynamics by the Rho family of GTPases, and the transduction of extracellular signals through focal adhesion complexes. Activation of the p38 mitogen-activated protein kinase and transforming growth factor- $\beta 1$ causes podocyte apoptosis. Phosphoinositide 3kinase and its downstream target AKT protect podocytes
\end{abstract}

This Minireview is based on an invited lecture given as part of the Takis Anagnostopoulos Symposium on Renal Physiology, Paris, June 26-27, 2008.

\section{KARGER}

๑ 2009 S. Karger AG, Basel

Fax +41613061234

E-Mail karger@karger.ch

www.karger.com
Accessible online at:

www.karger.com/nep from apoptosis. In human immunodeficiency virus-associated nephropathy, Src-dependent activation of Stat3, mitogen-activated protein kinase 1,2, and hypoxia-inducible factor $2 \alpha$ is an important driver of podocyte proliferation. At the level of intracellular signaling, it appears that different extracellular signals can converge onto a few pathways to induce changes in the phenotype of podocytes.

Copyright $\odot 2009$ S. Karger AG, Basel

\section{Introduction}

The visceral glomerular epithelial cell, also known as the podocyte, is a terminally differentiated, specialized cell with interdigitating foot processes (FPs) that wrap around the glomerular capillary tuft to form an integral component of the glomerular filtration barrier. Podocyte injury and loss have been observed in human and experimental models of glomerular diseases, including minimal change disease, focal segmental glomerulosclerosis (FSGS), membranous glomerulopathy, diabetic nephropathy (DNP), human immunodeficiency virus-associated nephropathy (HIVAN), and lupus nephritis [1]. Podocyte injury causing a reduction in the density of the podocyte is thought to lead to the progression of glomerulosclerosis and the eventual loss of renal function [2]. Studies from the past two decades have defined three major pathologic phenotypes exhibited by podocytes in glomerular dis-

Peter Y. Chuang

Box 1243, Division of Nephrology, Mount Sinai School of Medicine

One Gustave L. Levy Pl.

New York, NY 10029 (USA)

Tel. +1 212241 8004, Fax +1 212987 0389, E-Mail cijiang.he@mssm.edu 
eases: effacement, apoptosis, and proliferation. In this article, we intend to highlight the signaling cascades that are responsible for the manifestation of these pathologic phenotypes.

\section{Effacement}

The podocyte FP is delineated by three membrane domains: the apical membrane domain, the slit diaphragm (SD) protein complex, and the basal membrane domain [3]. The submembranous regions of all three compartments are linked to each other through the actin cytoskeleton. Disruption of any of the three domains or the underlying actin cytoskeleton can lead to FP effacement and disruption of the glomerular filtration barrier. Reorganization of the FP actin cytoskeleton appears to be a 'common final pathway' in FP effacement. This observation is not unexpected since several components of the SD complex (nephrin, zona occludens-1, and CD2AP) and focal adhesion complexes of the glomerular basement membrane (GBM; dystroglycans and integrins) are known to interact directly or indirectly with the actin cytoskeleton. The signaling molecules and pathways involved in the regulation of podocyte actin cytoskeleton were recently reviewed by Faul et al. [4]. Here, we highlight the interaction of nephrin with proteins in the SD complex, the regulation of actin dynamics by the Rho family of GTPases, and the transduction of extracellular signals through focal adhesion complexes, which are important for actin cytoskeleton organization and podocyte FP effacement.

\section{The Role of Nephrin as a Transducer of Extracellular}

Signals to the Actin Cytoskeleton

Nephrin is a transmembrane protein of the immunoglobulin superfamily. The extracellular immunoglobulin domains of nephrin interact with nephrin molecules from adjacent FP. The cytoplasmic tail of nephrin binds to intracellular adaptor proteins such as CD2AP, Nck2, and densin [5]. These adapter proteins interact directly with actin or indirectly through actin-binding proteins. The importance of nephrin on glomerular function is evident in humans with congenital nephrotic syndrome of the Finnish type, which is caused by mutations of the nephrin gene. Furthermore, a reduction in the expression of nephrin has been observed in several human and experimental proteinuric kidney diseases, suggesting that nephrin is essential for normal glomerular function.

Several studies have demonstrated that disruption of the proteins in the SD complex (i.e. podocin, TRPC6,
Neph1-3, and FAT) or proteins that interact with the SD complex (i.e. CD2AP, Nck, ZO-1, synaptopodin) can lead to the effacement of FP [4]. Nephrin has been described to act as a 'signaling node' in the SD by transmitting extracellular signals from the SD to the intracellular actin cytoskeleton [4]. For instance, the intracellular domain of nephrin contains six tyrosine residues that are conserved between human, mouse and rat. When phosphorylated, some of these tyrosine residues could serve as docking sites for SH2 domain-containing kinases and adaptor proteins [6]. Tyrosine phosphorylation of nephrin is dependent on its interaction with a number of nephrinbinding proteins, which stabilize nephrin at the SD and coordinate nephrin signaling [7]. Specifically, phosphorylation of nephrin has been shown to regulate FP morphology and actin dynamics through the Nck adaptor proteins [8]. Nck proteins contain one $\mathrm{SH} 2$ and three $\mathrm{SH} 3$ domains. The SH2 domain of Nck binds to phospho-tyrosine residues on nephrin, and the $\mathrm{SH} 3$ domain recruits other proteins involved in actin cytoskeleton regulation. It is thought that Nck and its associated actin cytoskeleton regulatory proteins are recruited to the phosphorylated nephrin when rapid actin polymerization and cytoskeletal reorganization is required during development and injury repair [8]. However, in steady state, nephrinNck interactions might be low and nephrine-CD2AP interactions predominate [6]. CD2AP is an adaptor protein that binds to nephrin and interacts with actin and actinbinding proteins [9]. These interactions provide a framework where extracellular signals could be transduced through CD2AP, nephrin, and actin-binding proteins to alter actin nucleation and FP organization [10].

\section{Modulation of Rho GTPases Activity on Actin \\ Cytoskeleton Dynamics}

Recently, two proteins - synaptopodin and diaphanous interacting proteins (DIPs) - were shown to modulate the activity of Rho in podocytes [11, 12]. The Rho family of GTPases is known to play an important role in the regulation of actin cytoskeleton dynamics and cell morphology in response to extracellular signals. The Rho and Rac subfamily members mediate the formation of actin stress fibers and membrane ruffles. These GTPases exist in either the GDP-bound inactive state or the GTPbound active state. The GTP-GDP exchange reactions and the activity of Rho GTPases are regulated by guanine nucleotide exchange factors (GEFs) and GTPase-activating proteins (GAPs). GEFs release GDP from Rho GTPases promoting the binding of GTP and activation of Rho GTPases. GAPs stimulate the intrinsic GTPase ac- 
tivity of Rho GTPases to catalyze the hydrolysis of GTP to GDP and inactivate Rho GTPases. In addition to GEFs and GAPs, synaptopodin also functions as an important modulator of RhoA activity in the podocyte [11]. Synaptopodin is a member of a class of proline-rich, actinassociated proteins that are found in the dendritic spine of neurons and podocyte FPs. Synaptopodin inhibits Smurf-1-mediated ubiquitination and subsequent proteasomic degradation of RhoA [11]. Recent studies from our laboratory delineated the role of DIP, a regulator of Rho and Rac signaling, on actin cytoskeleton dynamics in the podocyte. The human immunodeficiency virus (HIV) protein Nef interacts with DIP to increase Src-mediated phosphorylation of Vav2 and RhoA GAP, which are responsible for the loss of RhoA-mediated stress fiber formation and the increase in Racl-mediated lamellipodia formation and membrane ruffling observed in HIVAN [12].

\section{Podocyte-Basement Membrane Interactions}

Podocytes attach to the underlying GBM through two major cell adhesion complexes: $\alpha_{3} \beta_{1}$-integrin and $\alpha-/ \beta$ dystroglycans. The actin cytoskeleton interacts indirectly with integrins through integrin-associated proteins and directly with $\alpha$-/ $\beta$-dystroglycan. The importance of $\alpha_{3}$-integrin in podocyte function is demonstrated by the lack of FP formation in $\alpha_{3}$-deficient mice [13]. In podocytes, $\alpha_{3} \beta_{1-}$ integrin likely functions to modulate the podocyte cellmatrix adhesion rather than as an adhesion receptor since the lack of $\alpha_{3}$-integrin does not impair adhesion of podocytes but rather increases adhesion and protects against puromycin aminonucleoside-induced podocyte detachment [14]. The role of dystroglycans at the soles of the FP is uncertain, but observations suggest that they control the spacing of matrix proteins and possibly the porosity and permeability of the GBM [3]. In addition to their structural roles in the $\mathrm{FP}$ adhesion, integrins and dystroglycans are also transducers of extracellular signals in an 'outsidein' fashion to regulate intracellular actin dynamics [15]. They can also relay intracellular signals in an 'inside-out' manner to alter the adhesion of integrin to the GBM in response to intracellular events [15].

\section{Apoptosis}

Podocytes are terminally differentiated cells with a limited capacity to re-enter cell cycle and proliferate. Podocyte loss occurs in several glomerular diseases, including IgA nephropathy, lupus nephritis, FSGS, and
DNP [1]. In the case of DNP, a reduction in podocyte density is a critical determinant for the development of proteinuria and the progression of kidney dysfunction in diabetic patients [16]. Recent studies using murine models of diabetes mellitus suggest that the apoptosis of podocytes in these animals leads to a reduction in the density of podocytes [17]. Podocyte apoptosis has also been demonstrated in rats with puromycin-induced nephropathy [18], and transgenic mice expressing transforming growth factor (TGF)- $\beta 1$ [19]. The activation of p38 mitogen-activated protein kinase (MAPK) and TGF- $\beta 1$ are two well-described signaling pathways that mediate podocyte apoptosis (fig. 1). Activation of phosphoinositide 3-kinase (PI3K) and its downstream target AKT (protein kinase $\mathrm{B}$ ) protects against podocyte injury/apoptosis.

\section{Activation of p38 MAPK in Podocytes Causes Apoptosis}

Activation of the proapoptotic $\mathrm{P} 38 \mathrm{MAPK}$ pathway has been demonstrated in several animal models of glomerular diseases, including puromycin-induced nephrosis [18], crescentic glomerulonephritis [20], TGF- $\beta 1$ transgenic mice [21], and DNP [22]. MAPKs are major intracellular signal transduction factors mediating the transfer of extracellular stimuli to the nucleus. The MAPK activation cascade consists of three sequentially activated protein kinases. The $\mathrm{p} 38 \mathrm{MAPK}$ is activated by hyperosmolarity, oxidative stress and inflammatory cytokines. Activation of p38 leads to the phosphorylation of downstream targets and also the activation of nuclear transcription factors that are involved in apoptosis response. In animal models of DNP, activation of p38 by hyperglycemia through generation of reactive oxygen species has been shown to cause podocyte apoptosis [17]. Recently, we found that advanced glycation end products, which are known to play a pathogenic role in the development of DNP, triggered podocyte to undergo apoptosis through a p38-dependent pathway [23].

\section{Role of TGF- $\beta$ in Podocyte Apoptosis}

TGF- $\beta$ is a cytokine that has been shown to accumulate in injured kidneys of experimental animals and many types of chronic renal disease in humans. The TGF- $\beta$ isoforms are widely expressed in mammalian cells. They act on virtually all cell types in mammals by binding to the type II and I receptors and activate downstream Smad family proteins. Phosphorylated Smad proteins form a complex with Smad4 and translocate into the nucleus to activate the transcription of TGF- $\beta$ target gene. Trans- 


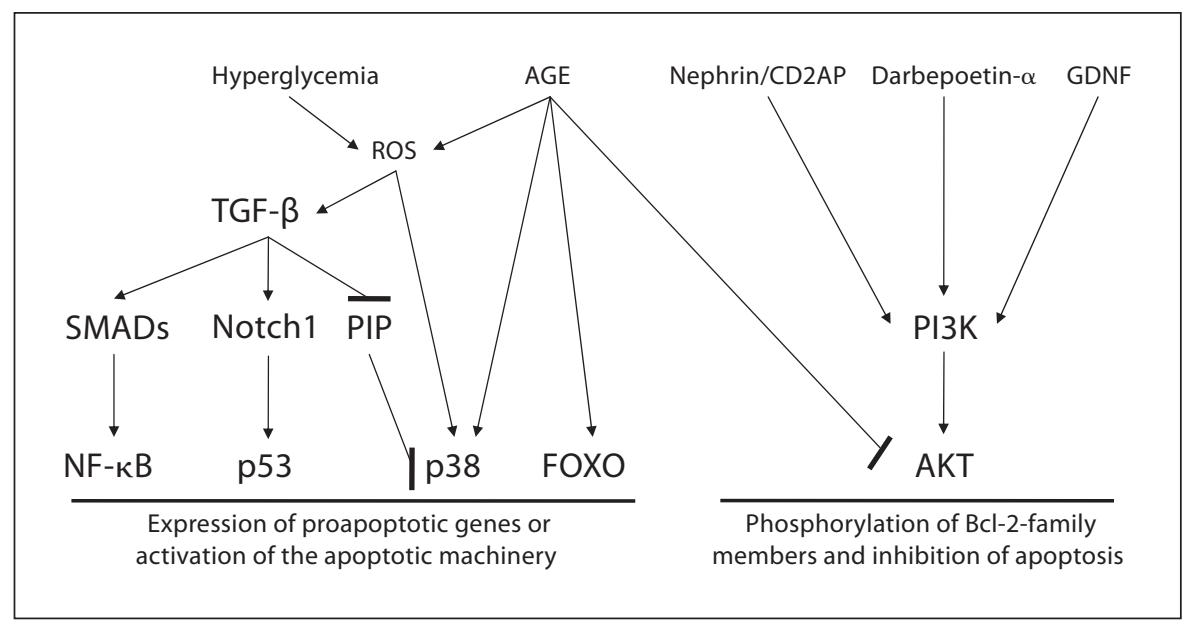

Fig. 1. Signaling in podocyte apoptosis. Activation of p38 and TGF- $\beta$ signaling pathways leads to the apoptosis of podocytes. TGF- $\beta$ is activated in podocytes by reactive oxygen species (ROS), which are known to accumulate in podocytes exposed to advanced glycation end products and hyperglycemia. The level of TGF- $\beta$ is increased in injured kidneys of several chronic kidney diseases. TGF- $\beta$ increases the expression of proapoptotic genes by activating transcription factors such as NF- $\mathrm{KB}$ and $\mathrm{p} 53$. Disruption of the PINCH-1-integrin-linked kinase and $\alpha$-parvin com- plex (PIP) by TGF- $\beta$ activates p38 [21]. Phosphorylation of p38 leads to the activation of the apoptosis machinery in podocytes. Advanced glycation end products (AGE) activate p38 and trigger the apoptosis of podocytes in a FOXO4-dependent manner [23]. AGE also reduces AKT phosphorylation. Activation of the PI3K/ AKT pathway by nephrin/CD2AP, darbepoetin- $\alpha$, and glialderived neurotrophic factor (GDNF) protects podocytes from apoptosis. genic mice overexpressing TGF- $\beta 1$ develop podocyte apoptosis and glomerulosclerosis through a p38MAPKand Smad7-mediated process [19]. Niranjan et al. [24] identified a novel mechanism of Notch1-dependent podocyte apoptosis in murine models of DNP and FSGS. They found that Notch1-dependent activation of p53, but not $\mathrm{p} 38 \mathrm{MAPK}$, is critical for TGF- $\beta 1$-induced apoptosis in these two animal models.

\section{AKT Is a Protective Signaling Pathway against \\ Podocyte Apoptosis}

Activation of the PI3K/AKT pathway by nephrin protected against detachment-induced apoptosis of cultured murine podocytes [25]. The antiapoptotic effect of AKT on podocyte apoptosis is further supported by the observation that a failure to phosphorylate AKT causes apoptosis of podocytes in $d b / d b$ mice [26], in oxidized LDLinduced podocyte injury [27], and in CD2AP-deficient mice [28]. The protective effect of darbepoetin- $\alpha$ [29] and glial cell-derived neurotrophic factor [30] against podocyte apoptosis in TGF- $\beta 1$-mediated and UV-induced podocyte apoptosis, respectively, is also dependent on the phosphorylation of AKT by PI3K. Using human embryonic kidney cells (293T) expressing podocyte SD complex proteins, nephrin, podocin and CD2AP, Huber et al. [25] showed that nephrin and CD2AP interact with PI3K and stimulate PI3K-dependent AKT signaling. However, there is no direct evidence to date to conclusively demonstrate that activation of the PI3K/AKT pathway by nephrin or $\mathrm{CD} 2 \mathrm{AP}$ in the podocyte protects against apoptosis.

\section{Proliferation and Dedifferentiation}

Podocytes are terminally differentiated, post-mitotic cells that, under normal conditions, have lost their ability to proliferate. Normal mature podocytes remain in a quiescent state and express cyclin-dependent kinase inhibitors p27 and p57 and do not express markers of proliferation (cyclin A, cyclin D, and Ki-67). However, in two specific podocyte diseases - HIVAN and idiopathic collapsing FSGS - podocytes exhibit hypertrophy as well as hyperplasia [31]. We found that Src-dependent activation of Stat 3 and MAPK1,2 pathways is a key driver of podocyte proliferation in HIVAN [32] (fig. 2). Recently, we also identified hypoxia inducible factor (HIF)-2 $\alpha$ as a downstream target of the Src-Stat3 pathway that mediates the proliferation of podocytes [33]. 


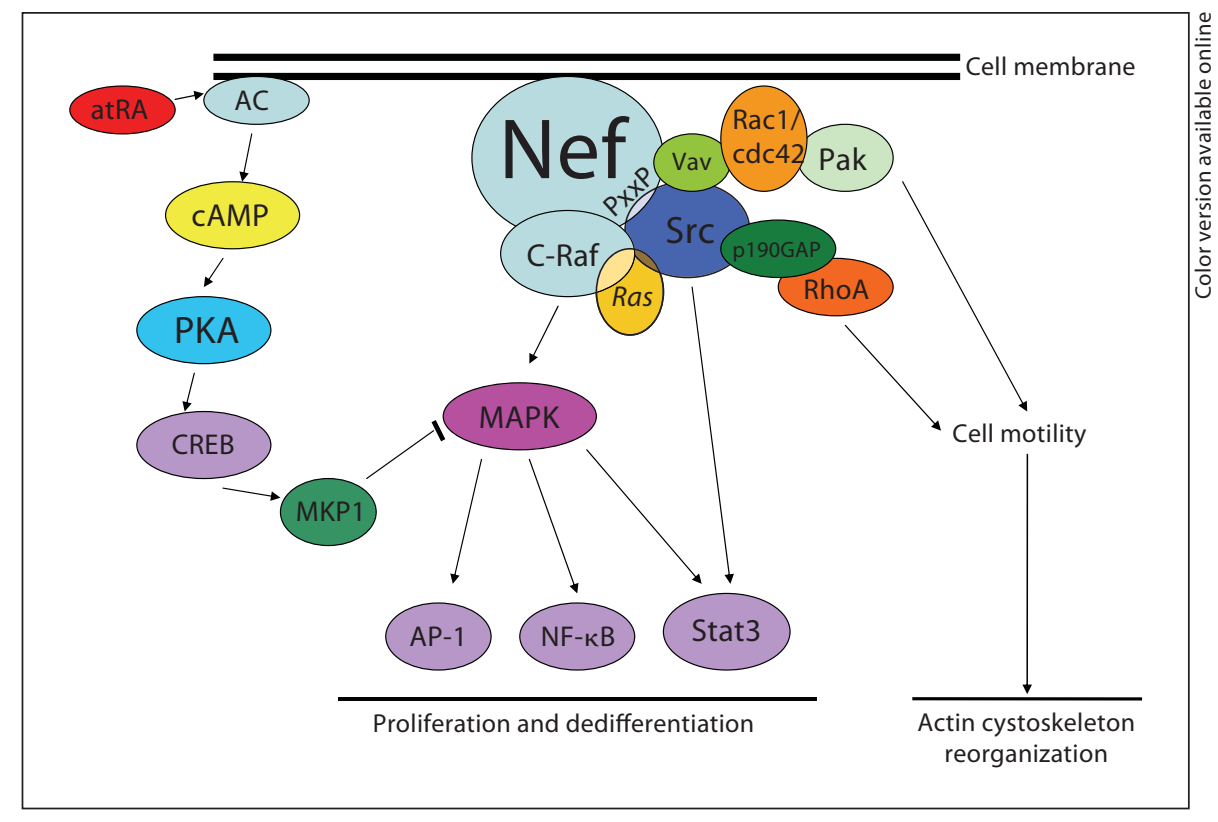

Fig. 2. Signaling pathways of podocyte proliferation in HIVAN. The HIV protein Nef induces cytoskeleton changes and cell proliferation/dedifferentiation in HIV-infected podocytes [12, 32]. Nef interacts with the Src family kinases through a prolinerich domain (PxxP). Nef inactivates RhoA by Src-mediated p190RhoAGAP phosphorylation to reduce stress fiber formation. Nef-mediated activation of Src also increases Vav2 phosphoryla- tion, which is responsible for Rac-induced lamellipodia formation. Src activation in Nef-expressing cells also induces the Ras-RafMAPK1,2 and Stat3 pathways to increase podocyte proliferation and dedifferentiation. atRA reverses the effects of HIV infection in podocytes by inhibiting Nef-mediated activation of MAPK1,2 through activation of $\mathrm{MKP} 1 . \mathrm{AC}=$ Adenyl cyclase; $\mathrm{CREB}=\mathrm{cAMP}$ response element-binding proteins; AP-1 = activator protein 1 .

\section{Role of Src, Stat3, and MAPK1,2 in Podocyte Proliferation}

The HIV protein Nef mediates the proliferation and de-differentiation of podocytes through Src-dependent activation of Stat3 and MAPK1,2 pathways [32]. The Src family kinases are key stimulators of cell proliferation, cell-cell adhesion, and cell motility [34]. These nonreceptor tyrosine kinases mediate these effects by protein phosphorylation, which then in turn activates signaling pathways and other protein-protein interactions. Members of the Src family kinases include Src, Hck, Fgr, Lck, Lyn, and Yes. Studies have implicated several of these kinases in podocyte pathophysiology. In HIVAN, we demonstrated that activation of the Src family kinases leads to podocyte proliferation and abnormal cytoskeleton structure in a Stat3- and MAPK1,2-dependent fashion [32]. Stat3 is activated in developing kidney and renal cell carcinoma. Phosphorylated Stat 3 translocates to the nucleus and activates the transcription of genes involved in cell growth, differentiation, and inflammation. Activation of the MAPK family plays a role in mitogenesis and cell differentiation. We showed that inhibition of Src activation pre- vented podocyte proliferation and cell dedifferentiation, a characteristic finding in collapsing FSGS of HIVAN [32].

\section{HIF-2 $\alpha$ and Vascular Endothelial Growth Factor \\ Pathway}

HIFs are a family of transcription factors composed of a heterodimer of $\alpha$ - and $\beta$-subunits that respond to changes in available oxygen in the cellular environment. The $\alpha$-subunit of HIF is degraded at normoxia by a process of von Hipple-Lindau protein (pVHL)-mediated ubiquitin-proteasome pathway. Under hypoxia condition, pVHL-mediated degradation of HIF- $\alpha$ is blocked, leading to transcriptional induction of HIF target genes, including vascular endothelial growth factor (VEGF). VEGF belongs to a family of angiogenic growth factors and plays a critical role in the maintenance of the glomerular filtration barrier [35]. Podocytes of transgenic mice with either podocyte-specific expression of VEGF [35] or podocyte-specific deletion of the von Hippel-Lindau gene Vhlh [36] develop a proliferative phenotype similar to what is seen in HIVAN. In the kidneys of patients with HIVAN as well as kidneys from HIV-1-trans- 
genic mice, the expression of VEGF and HIF- $2 \alpha$ are increased when compared with controls [33]. We found that in HIVAN activation of the Src-Stat3 pathway increases HIF-2 $\alpha$ and VEGF [33]. VEGF has been described by Foster et al. [37] to protect cultured human podocytes from serum starvation-induced apoptosis in a nephrin-dependent fashion. The increased activation of the HIF/VEGF pathway in HIV-infected podocytes might be a protective response against apoptosis in face of viral infection; however, it might also contribute to the uncontrolled proliferation of podocytes by promoting the survival of these diseased cells.

\section{All-Trans-Retinoic Acid Reduces Podocyte Proliferation}

Our studies on the effects of all-trans-retinoic acid (atRA) on HIV-induced podocyte proliferation suggest that activation of the CAMP pathway may have a protective effect against podocyte injury [38]. We found that the beneficial effects of atRA are mediated through the activation of cAMP-protein kinase A (PKA) pathway and reduction of MAPK1,2 phosphorylation. The cross-talk between the PKA and MAPK1,2 pathways in podocytes is through the activation of MAPK1,2 phosphatase 1 (MKP1). The expression of MKP1 is induced by atRA in a PKA-dependent manner. PKA increases the binding of cAMP response element-binding proteins and upstream transcription factor 1 (USF1) to the promoter regions of MKP1 to induce its transcription [39]. The suppression MAPK1,2 phosphorylation/activation by atRA appears to modulate the proliferative podocyte phenotype in HIVAN and reverts the podocytes back to a differentiated, nonproliferating phenotype in vitro [31]. The role of MKP1 in podocyte injury, however, needs to be confirmed in vivo.

\section{Conclusions}

The integrity of podocyte is essential for the maintenance of glomerular filtration barrier. The cellular response of podocytes to a wide variety of injuries in different glomerular diseases appears to be limited to three phenotypes: FP effacement, apoptosis, and proliferation. These changes, however, are not mutually exclusive. They can occur concurrently or progress from effacement to apoptosis or proliferation. At the level of intracellular signaling, different extracellular signals can converge onto a few pathways to induce a change in the phenotype of the podocyte. The challenge for future studies is to confirm and identify key signaling pathways that are responsible for the pathologic phenotypic change of podocytes in human diseases. This approach could yield targets for novel therapy to prevent and reverse podocyte dysfunction in glomerular diseases.

\section{Acknowledgment}

J.C.H. is supported by NIH grant R01 DK078897.

\section{References}

1 Shankland SJ: The podocyte's response to injury: role in proteinuria and glomerulosclerosis. Kidney Int 2006;69:2131-2147.

2 Wiggins RC: The spectrum of podocytopathies: a unifying view of glomerular diseases. Kidney Int 2007;71:1205-1214.

-3 Kerjaschki D: Caught flat-footed: podocyte damage and the molecular bases of focal glomerulosclerosis. J Clin Invest 2001;108: 1583-1587.

-4 Faul C, Asanuma K, Yanagida-Asanuma E, Kim K, Mundel P: Actin up: regulation of podocyte structure and function by components of the actin cytoskeleton. Trends Cell Biol 2007;17:428-437.

5 Patrakka J, Tryggvason K: Nephrin - a unique structural and signaling protein of the kidney filter. Trends Mol Med 2007;13: 396-403.
6 Putaala H, Sainio K, Sariola H, Tryggvason
K: Primary structure of mouse and rat neph-
rin CDNA and structure and expression of
the mouse gene. J Am Soc Nephrol 2000;11:
991-1001.
7 Huber TB, Benzing T: The slit diaphragm:
a signaling platform to regulate podocyte
function. Curr Opin Nephrol Hypertens
2005;14:211-216.

8 Verma R, Kovari I, Soofi A, Nihalani D, Patrie K, Holzman LB: Nephrin ectodomain engagement results in Src kinase activation, nephrin phosphorylation, Nck recruitment, and actin polymerization. J Clin Invest 2006; 116:1346-1359.

-9 Lehtonen S, Zhao F, Lehtonen E: CD2-associated protein directly interacts with the actin cytoskeleton. Am J Physiol Renal Physiol 2002;283:F734-F743.
10 Weaver AM, Young ME, Lee WL, Cooper JA: Integration of signals to the Arp2/3 complex. Curr Opin Cell Biol 2003;15:23-30.

11 Asanuma K, Yanagida-Asanuma E, Faul C, Tomino Y, Kim K, Mundel P: Synaptopodin orchestrates actin organization and cell motility via regulation of RhoA signalling. Nat Cell Biol 2006;8:485-491.

12 Lu TC, He JC, Wang ZH, Feng X, FukumiTominaga T, Chen N, Xu J, Iyengar R, Klotman PE: HIV-1 Nef disrupts the podocyte actin cytoskeleton by interacting with diaphanous interacting protein. J Biol Chem 2008;283:8173-8182.

13 Kreidberg JA, Donovan MJ, Goldstein SL, Rennke H, Shepherd K, Jones RC, Jaenisch R: Alpha 3 beta 1 integrin has a crucial role in kidney and lung organogenesis. Development 1996;122:3537-3547. 
- 14 Reiser J, Oh J, Shirato I, Asanuma K, Hug A, Mundel TM, Honey K, Ishidoh K, Kominami E, Kreidberg JA, Tomino Y, Mundel P: Podocyte migration during nephrotic syndrome requires a coordinated interplay between cathepsin 1 and alpha3 integrin. J Biol Chem 2004;279:34827-34832.

15 Blattner SM, Kretzler M: Integrin-linked kinase in renal disease: connecting cell-matrix interaction to the cytoskeleton. Curr Opin Nephrol Hypertens 2005; 14:404-410.

- 16 Steffes MW, Schmidt D, McCrery R, Basgen JM: Glomerular cell number in normal subjects and in type 1 diabetic patients. Kidney Int 2001;59:2104-2113.

- 17 Susztak K, Raff AC, Schiffer M, Bottinger EP: Glucose-induced reactive oxygen species cause apoptosis of podocytes and podocyte depletion at the onset of diabetic nephropathy. Diabetes 2006;55:225-233.

- 18 Koshikawa M, Mukoyama M, Mori K, Suganami T, Sawai K, Yoshioka T, Nagae T, Yokoi H, Kawachi H, Shimizu F, Sugawara A, Nakao K: Role of p38 mitogen-activated protein kinase activation in podocyte injury and proteinuria in experimental nephrotic syndrome. J Am Soc Nephrol 2005;16:26902701.

-19 Schiffer M, Bitzer M, Roberts IS, Kopp JB, ten Dijke P, Mundel P, Bottinger EP: Apoptosis in podocytes induced by TGF-beta and Smad7. J Clin Invest 2001;108:807-816.

-20 Stambe C, Atkins RC, Hill PA, Nikolic-Paterson DJ: Activation and cellular localization of the p38 and JNK MAPK pathways in rat crescentic glomerulonephritis. Kidney Int 2003;64:2121-2132.

-21 Jung KY, Chen K, Kretzler M, Wu C: TGFbetal regulates the pinch-1-integrin-linked kinase-alpha-parvin complex in glomerular cells. J Am Soc Nephrol 2007;18:66-73.

-22 Adhikary L, Chow F, Nikolic-Paterson DJ, Stambe C, Dowling J, Atkins RC, Tesch GH: Abnormal p38 mitogen-activated protein kinase signalling in human and experimental diabetic nephropathy. Diabetologia 2004;47: $1210-1222$.
23 Chuang PY, Yu Q, Fang W, Uribarri J, He JC: Advanced glycation endproducts induce podocyte apoptosis by activation of the FOXO4 transcription factor. Kidney Int 2007;72:965-976.

24 Niranjan T, Bielesz B, Gruenwald A, Ponda MP, Kopp JB, Thomas DB, Susztak K: The Notch pathway in podocytes plays a role in the development of glomerular disease. Nat Med 2008;14:290-298.

25 Huber TB, Hartleben B, Kim J, Schmidts M, Schermer B, Keil A, Egger L, Lecha RL, Borner C, Pavenstadt H, Shaw AS, Walz G, Benzing T: Nephrin and CD2AP associate with phosphoinositide $3-\mathrm{OH}$ kinase and stimulate AKT-dependent signaling. Mol Cell Biol 2003;23:4917-4928.

-26 Tejada T, Catanuto P, Ijaz A, Santos JV, Xia X, Sanchez P, Sanabria N, Lenz O, Elliot SJ, Fornoni A: Failure to phosphorylate AKT in podocytes from mice with early diabetic nephropathy promotes cell death. Kidney Int 2008;73:1385-1393.

27 Bussolati B, Deregibus MC, Fonsato V, Doublier S, Spatola T, Procida S, Di Carlo F, Camussi G: Statins prevent oxidized LDL-induced injury of glomerular podocytes by activating the phosphatidylinositol 3-kinase/AKT-signaling pathway. I Am Soc Nephrol 2005;16:1936-1947.

28 Schiffer M, Mundel P, Shaw AS, Bottinger EP: A novel role for the adaptor molecule $\mathrm{CD} 2$-associated protein in transforming growth factor-beta-induced apoptosis. J Biol Chem 2004;279:37004-37012.

29 Logar CM, Brinkkoetter PT, Krofft RD, Pippin JW, Shankland SJ: Darbepoetin alfa protects podocytes from apoptosis in vitro and in vivo. Kidney Int 2007;72:489-498.

30 Tsui CC, Shankland SJ, Pierchala BA: Glial cell line-derived neurotrophic factor and its receptor ret is a novel ligand-receptor complex critical for survival response during podocyte injury. J Am Soc Nephrol 2006;17: 1543-1552.
$31 \mathrm{Lu}$ TC, He JC, Klotman PE: Podocytes in HIV-associated nephropathy. Nephron Clin Pract 2007;106:c67-c71.

-32 He JC, Husain M, Sunamoto M, D’Agati VD, Klotman ME, Iyengar R, Klotman PE: Nef stimulates proliferation of glomerular podocytes through activation of Src-dependent Stat3 and MAPK1,2 pathways. J Clin Invest 2004;114:643-651.

- 33 Korgaonkar SN, Feng X, Ross MD, Lu TC, D’Agati V, Iyengar R, Klotman PE, He JC: HIV-1 upregulates VEGF in podocytes. J Am Soc Nephrol 2008;19:877-883.

34 Martin GS: The hunting of the Src. Nat Rev Mol Cell Biol 2001;2:467-475.

-35 Eremina V, Sood M, Haigh J, Nagy A, Lajoie G, Ferrara N, Gerber HP, Kikkawa Y, Miner JH, Quaggin SE: Glomerular-specific alterations of VEGF-A expression lead to distinct congenital and acquired renal diseases. J Clin Invest 2003;111:707-716.

36 Ding M, Cui S, Li C, Jothy S, Haase V, Steer BM, Marsden PA, Pippin J, Shankland S, Rastaldi MP, Cohen CD, Kretzler M, Quaggin SE: Loss of the tumor suppressor Vhlh leads to upregulation of Cxcr4 and rapidly progressive glomerulonephritis in mice. Nat Med 2006;12:1081-1087.

-37 Foster RR, Saleem MA, Mathieson PW, Bates DO, Harper SJ: Vascular endothelial growth factor and nephrin interact and reduce apoptosis in human podocytes. Am J Physiol Renal Physiol 2005;288:F48-F57.

38 He JC, Lu TC, Fleet M, Sunamoto M, Husain M, Fang W, Neves S, Chen Y, Shankland S, Iyengar R, Klotman PE: Retinoic acid inhibits HIV-1-induced podocyte proliferation through the camp pathway. J Am Soc Nephrol 2007;18:93-102.

-39 Lu TC, Wang Z, Feng X, Chuang P, Fang W, Chen Y, Neves S, Maayan A, Xiong H, Liu Y, Iyengar R, Klotman PE, He JC: Retinoic acid utilizes CREB and USF1 in a transcriptional feed-forward loop in order to stimulate MKP1 expression in human immunodeficiency virus-infected podocytes. Mol Cell Biol 2008;28:5785-5794 